\title{
Continuously Parametrized Quantum Simulation of Molecular Electron-Transfer Reactions
}

\author{
Frank Schlawin $\odot,{ }^{1,2,{ }^{*}, \S}$ Manuel Gessner $\odot,{ }^{3,2, \dagger}$ Andreas Buchleitner, ${ }^{2,4,5,6, \ddagger}$ Tobias Schätz, ${ }^{2,4,5}$ and \\ Spiros S. Skourtis 7,4 \\ ${ }^{1}$ Clarendon Laboratory, University of Oxford, Parks Road, Oxford OX1 3PU, United Kingdom \\ ${ }^{2}$ Physikalisches Institut, Albert-Ludwigs-Universität Freiburg, Hermann-Herder-Straße 3, Freiburg 79104, \\ Germany \\ ${ }^{3}$ Laboratoire Kastler Brossel, ENS-Université PSL, CNRS, Sorbonne Université, Collège de France, \\ 24 Rue Lhomond, Paris 75005, France \\ ${ }^{4}$ Freiburg Institute for Advanced Studies, Albert-Ludwigs-Universität Freiburg, Albertstraße 19, Freiburg 79104, \\ Germany \\ ${ }^{5}$ EUCOR Center for Quantum Science and Quantum Computing, Albert-Ludwigs-Universität Freiburg, \\ Hermann-Herder-Straße 3, Freiburg 79104, Germany \\ ${ }^{6}$ Erwin Schrödinger International Institute for Mathematics and Physics, University of Vienna, Boltzmanngasse 9 \\ Vienna 1090, Austria \\ ${ }^{7}$ Department of Physics, University of Cyprus, P.O. Box 20537 Nicosia 1678, Cyprus
}

(Received 6 April 2020; revised 29 November 2020; accepted 23 December 2020; published 25 January 2021)

A comprehensive description of molecular electron-transfer reactions is essential for our understanding of fundamental phenomena in bioenergetics and molecular electronics. However, experimental studies of molecular systems in condensed-phase environments face difficulties in independently controlling the parameters that govern the transfer mechanism with high precision. We show that, instead, trapped-ion experiments allow us to reproduce and continuously connect vastly different regimes of molecular charge transfer through precise tuning of, e.g., the phonon temperature, electron-phonon interaction, and electronic coupling. Such a setting does not only allow us to reproduce widely used transport models, such as Marcus theory. It also provides access to transfer regimes that are unattainable for molecular experiments, while controlling and measuring the relevant observables on the level of individual quanta. Our numerical simulations predict an unconventional quantum transfer regime, featuring a transition from quantum adiabatic to resonance-assisted transfer as a function of the donor-acceptor energy gap, which can be reached by increasing the electronic coupling at low temperatures. Trapped-ion-based quantum simulations thus promise to enhance our microscopic understanding of molecular electron-transfer processes and may help to reveal efficient design principles for synthetic devices.

DOI: 10.1103/PRXQuantum.2.010314

\section{INTRODUCTION}

Molecular electron-transfer (ET) reactions are the fundamental steps in many chemical and biological processes [1-6]. A detailed understanding of the

\footnotetext{
*frank.schlawin@mpsd.mpg.de

†manuel.gessner@ens.fr

$\$$ abu@uni-freiburg.de

§Present address: Max-Planck-Institute for the Structure and Dynamics of Matter, Hamburg

Published by the American Physical Society under the terms of the Creative Commons Attribution 4.0 International license. Further distribution of this work must maintain attribution to the author(s) and the published article's title, journal citation, and DOI.
}

underlying physics is crucial in such diverse fields as catalysis [5], photosynthesis [7], biological signaling [8], molecular electronics [9], and energy conversion [10,11]. Marcus theory, the fundamental theory for thermally activated nonadiabatic ET rates, has already been developed in 1956 [1] and has since been generalized to include quantum or classical vibrations that modulate the energy gap or the coupling $[1-6,12]$. However, many ET reactions cannot be described by incoherent nonadiabatic rate theories. Currently, intensive research efforts are being dedicated to nonstandard transport regimes $[11,13,14]$. These include fast charge- and energy-transfer reactions on the time scales of vibrational relaxation and electronic dephasing, such as the primary reactions in photosynthesis [11], nonergodic and nonequilibrium vibrational dynamics [13, 
15], the transition from incoherent to coherent transport [16], the participation of quantum vibrations in promoting ultrafast ET, and the control of ET by external fields [15].

An understanding of the particulars of a molecular ET reaction calls for deciphering how the molecular assembly and dynamics determine the dominant transfer mechanism and the magnitude of the transfer rate. A common experimental approach is to chemically modify the molecular and/or solvent structure with the aim of changing some of the fundamental parameters that determine the transport mechanism (e.g., tunneling barrier heights, electron-phonon coupling strengths, etc.) $[3,13,14,17]$. It is challenging to achieve tunable and independent control of these parameters, as is best exemplified by the experimental efforts to verify Marcus theory. The theory predicts that the logarithmic ET rate has an inverse-parabolic dependence on the electron donor-to-acceptor $(D-A)$ energy gap $\left(\Delta E=E_{A}-E_{D}\right)$, if all other parameters are kept constant. As the energy gap is increased, the rate increases (normal region), reaches a maximum (activationless transfer), and then decreases (inverted region). The first experimental verification of the inverted region was accomplished almost 30 years after its theoretical prediction $[18,19]$ and led to the Nobel Prize for ET theory in 1992 [20]. In this seminal experiment, eight discrete distinct values of the energy gap were realized by synthesizing eight different acceptor species, attached to a donor by a rigid molecular bridge. Obviously, despite its spectacular success, such a strategy is unable to monitor the competition between distinct ET mechanisms, under continuous tuning of the system parameters that control the subtle interplay between direct electronic coupling and strong interactions with phononic baths.

An experimental platform that offers precisely this kind of continuous tunability consists of arrays of electrostatically interacting ions trapped by harmonic external potentials [21-24], with an accuracy of individual parameter control evidenced by their performance among the best atomic clocks [25]. These systems are currently considered among the leading architectures for the realization of quantum simulators and computers [22-24,26,27]. The relevant coupling strengths, energy scales, and dissipative processes are determined with high accuracy by laserand radio-frequency fields. The versatility of trapped-ion arrays renders them ideal candidates for the study of elementary quantum transport processes under controlled conditions. Previous studies have focused on the transport of electronic (effective spins) and vibrational excitations (phonons) across ion chains [28-31] and on the influence of spin-phonon coupling on coherent exciton transport processes [32].

Here, we show that analog trapped-ion quantum simulations with strong electron-phonon interactions allow us to continuously map out the parameter space of a paradigmatic ET scenario. We introduce and discuss a minimal setting readily accessible for state-of-the-art experiments that is able to reproduce the predictions of Marcus theory, including the nonadiabatic inverted regime. We further establish how lowering the temperature allows us to reach the nonadiabatic quantum transport regime, where vibronic (induced by the strong coupling of vibrational and electronic degrees of freedom) resonances determine the transfer rates. Finally, we demonstrate that the iontrap platform makes unconventional ET regimes accessible that are not observed in molecular systems. We predict the crossover from conventional nonadiabatic molecular ET to a quantum transfer regime where rates are limited by phonon lifetimes in the normal regime and heavily modulated by resonances in the inverted regime. These modulations can be understood as a consequence of trapped excited-state populations that do not participate in the adiabatic transport and only contribute to the transport on resonance.

Our paper is structured as follows. We start with an overview of the Hamiltonian and the phenomenology of ET in Sec. II. This is followed in Sec. III by an outline of the elementary experimental platform for proof-ofprinciple quantum simulations of ET models. In Secs. IV and $\mathrm{V}$, we then present simulations of this platform, where we demonstrate that it can access well-established molecular ET regimes as well as exotic new transport regimes that are not observed in molecular samples. We then conclude with an outlook on possible extensions in Sec. VI and a summary in Sec. VII.

\section{ELECTRON-TRANSFER THEORY}

ET from a donor $(D)$ to an acceptor state $(A)$ necessarily incorporates a large change of molecular charge distributions, such that the process is strongly coupled to the phononic degrees of freedom of the ET molecule. In its simplest form, such a process can be described by the Hamiltonian

$$
H=\hbar \omega_{0} a^{\dagger} a-\frac{\Delta E}{2} \sigma_{z}+V \sigma_{x}+\frac{\hbar g}{2} \sigma_{z}\left(a+a^{\dagger}\right),
$$

where the first term describes a phononic mode that facilitates the ET. It gives rise to a quadratic potential in the reaction coordinate $z=\left(a+a^{\dagger}\right) / 2$. The second term, with $\sigma_{z}=|D\rangle\langle D|-| A\rangle\langle A|$, adds a state-dependent energy shift $\pm \Delta E / 2$ of the vibrational harmonic oscillator potentials associated with the acceptor and donor levels, leading to a total energy offset $\Delta E$ (the donor-acceptor energy gap of the ET reaction) between donor and acceptor BornOppenheimer (BO) surfaces. Here, we adopt the definition $\Delta E=E_{A}-E_{D}$, which follows the convention of ET theory, where a negative $\Delta E$ expresses the fact that energy is released during the ET process [1-6]. The third term coherently couples the donor and acceptor states, through $\sigma_{x}=|A\rangle\langle D|+| D\rangle\langle A|$. It has the strongest impact on the 


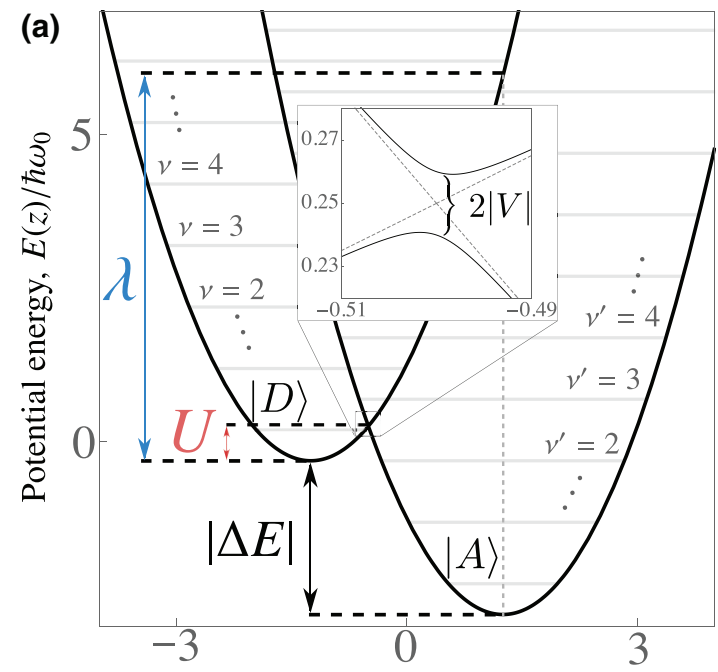

Oscillator position, $z=\left(a+a^{\dagger}\right) / 2$

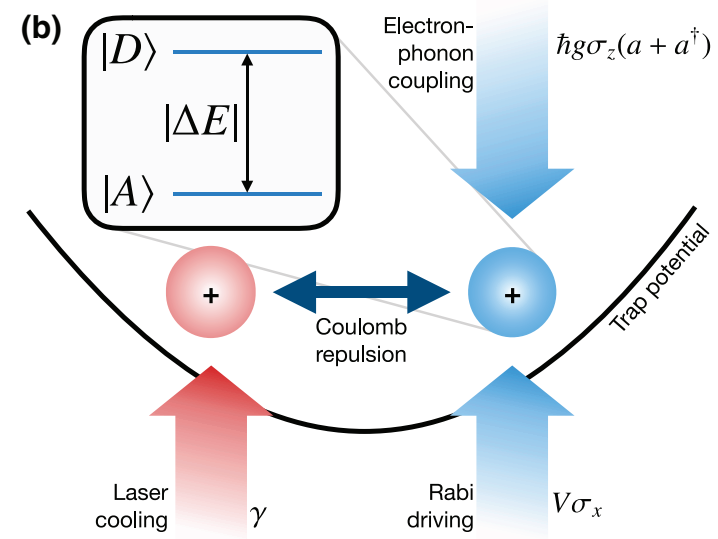

FIG. 1. Electron-transfer theory. (a) The vibrational-energy landscape deduced from the Hamiltonian (1), composed of the adiabatic Born-Oppenheimer (BO) surfaces for the electronic states $D$ and $A$, as a function of the reaction coordinate defined by the dimensionless harmonic oscillator position variable $z$. The diabatic BO surfaces, $E_{D}(z)$ (left parabola) and $E_{A}(z)$ (right parabola), respectively, are identified by dashed gray lines in the inset. For the weak coherent coupling assumed here, adiabatic and diabatic surfaces are indiscernible on the scale of the main plot. The $D-A$ energy gap $\Delta E=E_{A}-E_{D}$ [which is negative given the electronic level structure depicted in (b) below], the reorganization energy $\lambda$, the activation energy $U$, and the coherent coupling $V$ (inset) are indicated. The horizontal lines indicate vibrational levels $v\left(v^{\prime}\right)$ of the $D(A)$ BO surface upon quantization. The employed parameters are $|\Delta E|=2.5 \hbar \omega_{0}$, $V=0.01 \hbar \omega_{0}$, and $g=2.5 \omega_{0}$, where $\omega_{0}$ denotes the (effective) trap frequency. (b) A "system" ion (blue) simulates the donoracceptor system with the two electronic states $|D\rangle$ and $|A\rangle$ (akin to a spin 1/2). Laser-driven Rabi oscillations (blue upward arrow) induce coherent coupling of strength $V$ between the donor and acceptor states. A second laser (blue downward arrow) induces spin-phonon coupling between the electronic degree of freedom of the "system" ion and a collective motional mode of both ions. This mode is sympathetically cooled by laser interaction (red arrow) through a "cooling" ion (red) of a different species. vibrational-energy landscape at the degeneracy of the $\mathrm{BO}$ surfaces of the donor and the acceptor [see Fig. 1(a)], where it induces an avoided crossing of size $2|\mathrm{~V}|$, associated with a coherent population transfer at Rabi frequency $V / \hbar$. Finally, the fourth (coupling) term, proportional to $\sigma_{z}\left(a+a^{\dagger}\right)=2 \sigma_{z} z$, contributes a linear potential in the reaction coordinate $z$, with a positive or negative sign depending on whether the system resides in the donor or acceptor state $|D\rangle$ or $|A\rangle$. This displaces the harmonic oscillator vibrational potential minimum from the origin to $z_{1}=$ $-g / 2 \omega_{0}$ (donor) and $z_{2}=g / 2 \omega_{0}$ (acceptor), respectively.

Crucially, the vibrational mode is subject to fast dissipation caused by the coupling to environmental modes. The dynamics are therefore described by the master equation

$$
\partial \rho(t) / \partial t=-(i / \hbar)[H, \rho(t)]+\mathcal{D}[\rho(t)],
$$

where the Lindblad dissipator is given by a damped harmonic oscillator [33]

$$
\begin{aligned}
\mathcal{D}(\rho)= & \gamma(1+\bar{n})\left(a \rho a^{\dagger}-\frac{1}{2}\left\{a^{\dagger} a, \rho\right\}\right) \\
& +\gamma \bar{n}\left(a^{\dagger} \rho a-\frac{1}{2}\left\{a a^{\dagger}, \rho\right\}\right)
\end{aligned}
$$

and $\{A, B\}=A B+B A$.

To understand the ET process from donor to acceptor $(|D\rangle \rightarrow|A\rangle)$ governed by the open-system dynamics in Eq. (2), it is most intuitive to consider the potential landscape as a function of the reaction coordinate $z$, depicted in Fig. 1(a), with the BO surfaces $E_{D}(z)$ and $E_{A}(z)$. In molecular ET systems, the reaction coordinate is the displacement of a molecular vibrational mode (or a set of modes) whose equilibrium conformation is changed by the electronic transition from $|D\rangle$ to $|A\rangle$. A key parameter of the transport process is the reorganization energy $\lambda$. It is the energy required to distort the minimum-energy conformation of the molecule in the electronic state $|D\rangle$ to the minimum-energy conformation in the state $|A\rangle$, without switching state from $|D\rangle$ to $|A\rangle$. It is thus the energy needed to displace the reaction coordinate on the donor BO surface from its minimum position to that of the acceptor BO surface [1-6]. In the geometry determined by Eq. (1), this amounts to $\lambda=\hbar \omega_{0}\left(z_{1}-z_{2}\right)^{2}=\hbar g^{2} / \omega_{0}$. A large reorganization energy compared to the phonon frequency signifies that such displacement will be associated with strong vibrational excitations. The phonon mode is thus strongly coupled to the electronic degree of freedom and actively contributes to the ET reaction (i.e., it is ET active). This is in contrast to models of vibrationally assisted transport of excitations, where the coupling to phonons is typically less pronounced and the transport is predominantly coherent and therefore reversible [8].

Irreversible population transfer from $|D\rangle$ to $|A\rangle$ is mediated by classical or quantum-mechanical mechanisms, 
depending on the dominant energy scale. These are the thermal energy $k_{B} T$, the phonon energy $\hbar \omega_{0}$, the coupling strength $V$, the energy gap $\Delta E$, and the reorganization energy $\lambda$. On the one hand, the ratio of the temperature and the phonon energy determines whether the system is operated in a quantum or classical parameter regime. On the other hand, the relative (as compared to reorganization and phonon energy, respectively) strength of the avoided crossing of size $2|V|$ between the two $\mathrm{BO}$ surfaces controls the transition from nonadiabatic (between two distinct diabatic BO surfaces) to adiabatic (between the minima of the same lower BO surface) transfer. In this paper, we always consider cases where the initial and final electronic states are well localized in different regions of the molecule, known as localized polaron states with $|V|<\lambda / 4$ [1-6].

\section{TRAPPED-ION SETUP}

The ion-trap setup that we propose in order to simulate ET is sketched in Fig. 1(b). Two positively charged ions are electrostatically trapped in a harmonic potential and repel each other by the Coulomb force. By assuming only small displacements around the equilibrium positions, the motion of the two-ion compound can be described by six normal phonon modes (two in each dimension) [34]. The electronic quantum state of the ion can be controlled with resonant electromagnetic fields. Employing near-resonant fields where the detuning is chosen to match a resonance condition involving the phonon modes allows us to control the coupling between select electronic and vibrational degrees of freedom.

For the implementation of ET physics, the first, "system," ion (blue dot) emulates the donor and acceptor states $|D\rangle$ and $|A\rangle$, respectively, by means of two long-lived electronic levels [22-24,34]. These levels form an effective pseudospin, described by $-(\Delta E / 2) \sigma_{z}$, where $\Delta E$ is the associated resonance frequency, which can be controlled by an external magnetic field via the Zeeman effect. Driving the transition between these two levels with a resonant electromagnetic field induces Rabi oscillations, described by $V \sigma_{x}$, where $V / \hbar$ is the Rabi frequency [blue upward arrow in Fig. 1(b)]. The second, "cooling," ion (red dot) is used exclusively to sympathetically cool the collective motion of the two-ion compound [34-40]. Choosing spectrally distinguishable ions or employing tightly focused laser beams prevents the cooling laser light from affecting the internal states of the system ion. The Hamiltonian of the relevant normal phonon mode of frequency $\omega_{0}$, determined by the trap potential [34,41], is given by $\hbar \omega_{0} a^{\dagger} a$, and the laser-cooling evolution gives rise to the dissipator given in Eq. (3). Finally, the spin-phonon interaction term $\hbar g \sigma_{z}\left(a+a^{\dagger}\right) / 2$ between the vibrational and the electronic degree of freedom [blue downward arrow in Fig. 1(b)] can be realized by bichromatic laser driving with suitably chosen detuning close to the trap frequency [32,42-45]. The electron-phonon coupling constant $g$ is then determined via the laser intensities and detunings [32,34,45]. An implementation with traveling-wave light is also possible. It requires a rotating-frame description of the dynamics, which causes the trap frequency $\omega_{0}$ to be replaced by the laser detuning. Throughout this paper, we express all energy and time scales in units of $\omega_{0}$, which may represent an effective trap frequency. A similar spin-boson interaction term, where the coupling is given by $\sigma_{x}$ rather than $\sigma_{z}$, appears in the Rabi model, which can also be simulated with high flexibility in a trapped-ion setup using related methods [46]. Taken together, the effective dynamics of the electronic degree of freedom of the system ion, together with the collective vibrational degree of freedom of the compound, are formally identical to the standard models of ET theory in Eq. (1).

All relevant aspects of the dynamics can be discussed and experimentally demonstrated starting with two trapped ions, coupled by the Coulomb force while sharing the same trap potential. Extensions to larger numbers of ions or different trap geometries and dimensionalities [47] provide access to more complex situations that involve a larger number of electronic or phononic modes, giving rise, e.g., to intermediate bridge states [48].

In contrast to real molecular systems, all these relevant system parameters are continuously and precisely tunable over wide intervals. We summarize the correspondence

TABLE I. The correspondence between the characteristic parameters of electron transfer (left) and their counterparts in trapped-ion simulations (center), together with typical experimental values in ion-trap platforms (right). The notation in the left column is adopted from Ref. [6].

\begin{tabular}{lll}
\hline \hline Molecular electron transport & Ion-trap simulation & Parameter range \\
\hline Reaction energy gap $\Delta E$ & Zeeman splitting $\Delta E$ & $|\Delta E| / \hbar \sim B \times 5-25 \mathrm{kHz} / \mu \mathrm{T}[49]$, \\
& & where $B \lesssim 30 \mathrm{mT}$ \\
Reorganization energy $\lambda$ & State-dependent dipole force $g \Rightarrow \lambda=\hbar g^{2} / \omega_{0}$ & $g \lesssim 2 \pi \times 500 \mathrm{kHz}[44]$ \\
Phonon energy $\omega$ & Trap frequency $\omega_{0}$, & $\omega_{0} \sim 2 \pi \times 0.05-10 \mathrm{MHz}[22,23,34,50]$ \\
& or laser detuning $\delta$ & $100 \mathrm{~Hz} \lesssim \delta \lesssim \omega_{0} / 2[32]$ \\
Electronic coupling $V$ & Rabi frequency $V / \hbar$ & $V / \hbar \lesssim 2 \pi \times 1 \mathrm{MHz}[22,23]$ \\
Resonance width $\Gamma$ & Sympathetic cooling rate $\gamma$ & $\gamma \lesssim 20 \mathrm{kHz}[34,51]$ \\
Thermal energy $k_{B} T$ & Average phonon number $\bar{n} \Rightarrow k_{B} T=\hbar \omega_{0} / \ln [(\bar{n}+1) / \bar{n}]$ & $\bar{n} \gtrsim 0.01[34,37,52-54]$ \\
\hline \hline
\end{tabular}


between the most important ET parameters and their analogs in the proposed trapped-ion simulation in Table I. In the following, we explore different transfer regimes by controlling individually the thermal energy $k_{B} T$, the (effective) trap frequency $\omega_{0}$, and the coupling strength $V$ in numerical simulations of the time evolution governed by Eq. (2).

\section{CLASSICAL NONADIABATIC TRANSPORT}

The classical regimes involve ET-active vibrational frequencies with $\hbar \omega_{0} \ll \lambda$ and temperatures with $\hbar \omega_{0} \ll$ $k_{B} T$. Many molecular ET reactions in condensed phases (i.e., in solution, cellular, or molecular-junction environments rather than in vacuum) belong to this category because low-frequency environmental vibrations couple to ET. In this regime, the Landau-Zener parameter $\gamma_{\mathrm{LZ}}=\pi^{3 / 2}|V|^{2} /\left(\hbar \omega_{0} \sqrt{\lambda k_{B} T}\right)$ distinguishes the nonadiabatic (weak electronic coupling, $\gamma_{\mathrm{LZ}} \ll 1$ ) and adiabatic (strong electronic coupling, $\gamma_{\mathrm{LZ}} \gtrsim 1$ ) limits [55].

The classical nonadiabatic case $(\mathrm{CN})$ is described by Marcus theory. Classical thermally activated vibrations can tune the electronic states $|D\rangle$ and $|A\rangle$ to resonance, such that ET can take place at the crossing point of the two diabatic BO surfaces, while preserving the total energy of the system. The ET rate is then given by the expression [1-6]

$$
k_{\mathrm{CN}}=\frac{2 \pi}{\hbar} \frac{|V|^{2}}{\sqrt{4 \pi \lambda k_{B} T}} \exp \left(-\frac{U}{k_{B} T}\right)
$$

where $U=(\Delta E+\lambda)^{2} /(4 \lambda)$ is the activation energy to the resonance conformation on the donor BO surface [see Fig. 1(a)]. This transfer rate is proportional to $|V|^{2}$ and reaches a maximum in the activationless limit $(U=0)$, when $|\Delta E|=\lambda$, such that the acceptor BO surface intersects the minimum of the donor surface [see Fig. 2(a), panel $\mathrm{C}]$. The dependence of $\ln k_{\mathrm{CN}}$ on the detuning $\Delta E$ is an inverted parabola - the seminal Marcus parabola, cf. the orange line in Fig. 2(c).

This parameter regime can be reached in the trapped-ion system for a thermal-phonon population with a relatively low $\omega_{0}$. For our numerical simulations of the trappedion dynamics, we initialize the system at time $t=0$ in $|D\rangle\left\langle D\left|\otimes \sum_{\nu} p_{v}\right| v\right\rangle\langle v|$, where $|v\rangle$ is a Fock state of $v$ phonons, weighted by a thermal distribution $p_{v}$ at temperature $T$. We extract the ET rate $k$ by fitting an exponential decay $a \exp (-k t)$ to the time evolution of the donor-state population $p_{D}(t)$ after a transient evolution of $\omega_{0} t / 2 \pi=5$ that is dominated by coherent dynamics (see also Appendix A). Typical evolutions are displayed in Fig. 2(b) for $k_{B} T \simeq 10.5 \hbar \omega_{0}$ and $\gamma_{\mathrm{LZ}} \simeq 10^{-3}$. The corresponding average phonon number of $\bar{n}=10$ can be reached without requiring efficient cooling. Figure 2(c) shows the extracted transfer rates from the trappedion simulation (dark blue circles). These follow almost
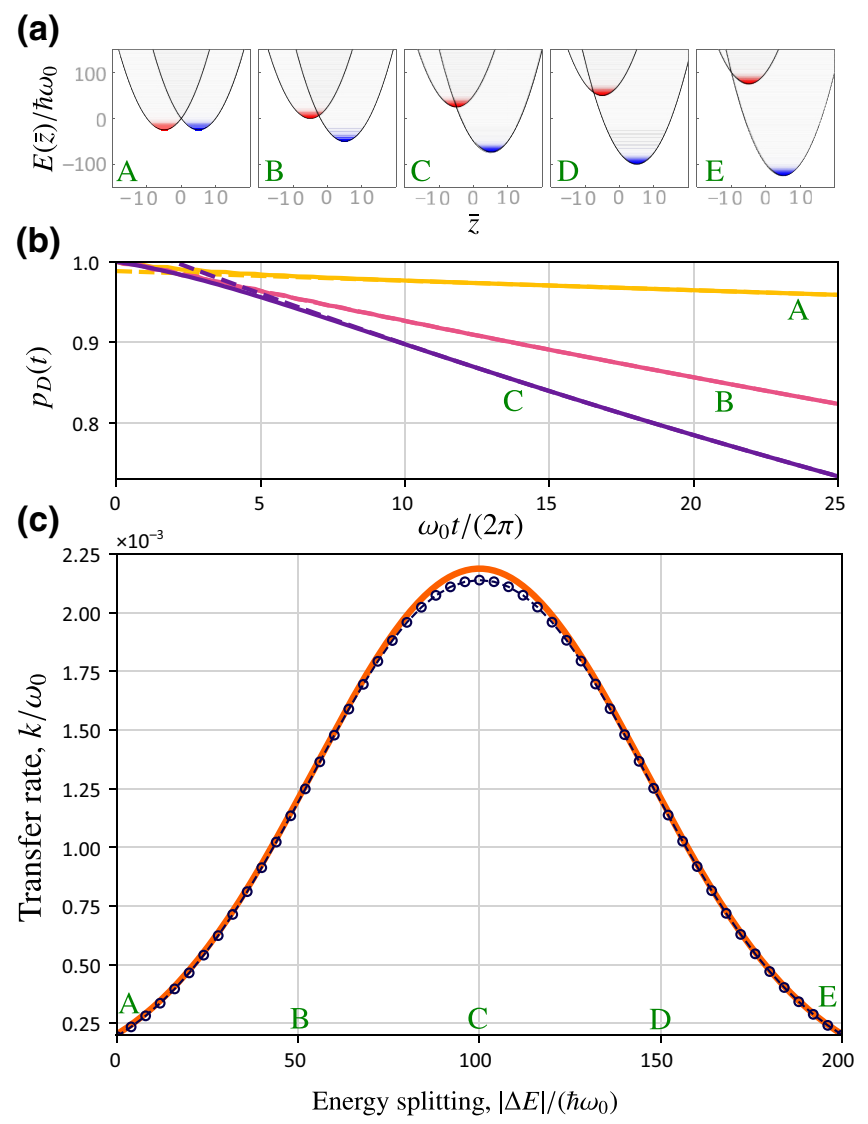

FIG. 2. Classical nonadiabatic transfer. (a) Diabatic BO energy surfaces and their vibrational levels in the classical nonadiabatic regime. The parameters are chosen as $V=0.2 \hbar \omega_{0}$, $g=10 \omega_{0}, \gamma=0.4 \omega_{0} / 2 \pi$, and $\bar{n}=10$, corresponding to $k_{B} T \approx$ $10.5 \hbar \omega_{0}$ and $\lambda=100 \hbar \omega_{0}$. The panels show $|\Delta E|=0 \hbar \omega_{0}$ (A), $|\Delta E|=50 \hbar \omega_{0}(\mathrm{~B}),|\Delta E|=100 \hbar \omega_{0}(\mathrm{C}),|\Delta E|=150 \hbar \omega_{0}$ (D), and $|\Delta E|=200 \hbar \omega_{0}(\mathrm{E})$. Vibrational states are indicated by horizontal gray lines and red (donor) and blue (acceptor) shaded areas indicate the thermal populations. (b) The exponential fit (dashed lines) of the time evolution of the donor-state population $p_{D}(t)$ (continuous lines) as generated by Eqs. (1) and (3), for the parameters of panels A (yellow), B (pink), and C (violet) in (a). (c) Transfer rates extracted from the exponential fits to $p_{D}(t)$ as a function of $|\Delta E|$ (dark blue circles). The orange line is the prediction of classical nonadiabatic transport theory, according to the Marcus formula (4).

perfectly the Marcus prediction (orange line), given in Eq. (4), which we plot without adjustable parameters. Both the normal and the inverted transport regimes can be simulated. Small deviations near the activationless case may be due to a broadening of the phonon resonances caused by the laser-cooling process, which is not captured by Marcus theory of Eq. (4).

\section{QUANTUM REGIMES}

Let us now turn to the discussion of quantum regimes for ET. When $k_{B} T \approx \hbar \omega_{0}$, the quantum nature of the vibrational mode has to be accounted for when modeling the 
transfer process. We first consider the parameter regime $|V| \ll \lambda / 4$, where $V$ and $\hbar \gamma$ are of the same order of magnitude, which is described by quantum nonadiabatic (QN) ET theory $[1,2,4,6]$. In Fig. 1(a), this situation is sketched by indicating the respective energies, $E_{D, \nu}$ and $E_{A, v^{\prime}}$, of the uncoupled donor and acceptor vibronic eigenstates $|D\rangle|v\rangle$ and $|A\rangle\left|v^{\prime}\right\rangle$ as horizontal lines. Population transfer is then facilitated by resonances between pairs of such states, as expressed by Fermi's golden rule $[1,2,4,6]$ :

$$
k_{\mathrm{QN}}=\frac{2 \pi}{\hbar}|V|^{2} \sum_{v, v^{\prime}} p_{v} \mathrm{FC}_{v, v^{\prime}} \delta\left(E_{D, v}-E_{A, v^{\prime}}\right)
$$

where the $p_{v}$ describe the initial thermally distributed phonon populations and $\mathrm{FC}_{v, v^{\prime}}=\left|\left\langle v\left|e^{g\left(a^{\dagger}-a\right) / \omega_{0}}\right| v^{\prime}\right\rangle\right|^{2}$ is the Franck-Condon factor between two displaced harmonic oscillators of identical frequency.

This ET transfer regime can be simulated on the iontrap platform with standard parameters, i.e., by cooling to smaller phonon numbers as compared to those discussed in the classical regime above. Figure 3(a) shows the vibronic energy levels in the quantum nonadiabatic regime for different values of $\Delta E$. The time evolution of the donorstate population is fitted to an exponential decay [after a transient evolution of $\omega_{0} t / 2 \pi=100$; see Fig. 3(b)] for $\bar{n}=0.01\left(k_{B} T \simeq 0.217 \hbar \omega_{0}\right)$. Figure $3(\mathrm{c})$ depicts the variation of the obtained transfer rates with $\Delta E$. The classical Marcus parabola is now heavily modulated by vibronic resonances, which allow for fast ET at sharply defined values of the donor-acceptor energy splitting. The transfer rates are extremely well described by the predictions of nonadiabatic theory, given in Eq. (5), which is plotted for comparison [56]. We note that this vibronic modulation of the resonances, which is readily accessible in an ion-trap quantum simulation, is washed out for molecular ET experiments in condensed-phase environments by uncontrolled disorder and additional coupling to lowfrequency vibrations. Figures 2 and 3 demonstrate that current ion-trap technology can simulate well-known ET rate regimes. As such, these results define a benchmark for the experimental ability to simulate the ET Hamiltonian.

With increasing electronic coupling $V$ (and all other parameters fixed)-which is controlled by the injected laser power - the above picture of resonant transitions between diabatic states is washed out by the opening of an appreciable avoided crossing between the donor and acceptor BO surfaces [see Fig. 4(a)], ultimately defining two new excited and ground-state adiabatic potential surfaces that no longer intersect.

This trend toward a dominant role of $V$ manifests in a marked change of the dependence of the transfer rate on the energy gap: As one enters the regime $|V| \gg \hbar \gamma$, strong direct coupling $V$ quickly transfers the initial population from the donor to the acceptor - where it can only remain if the phonons created in the processes are promptly (a)

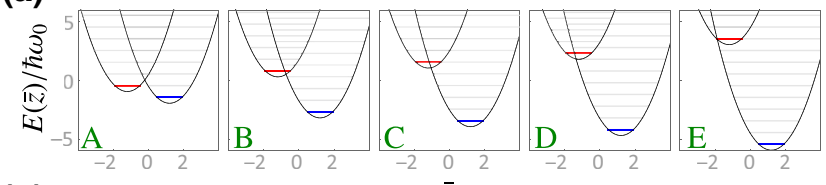

(b)
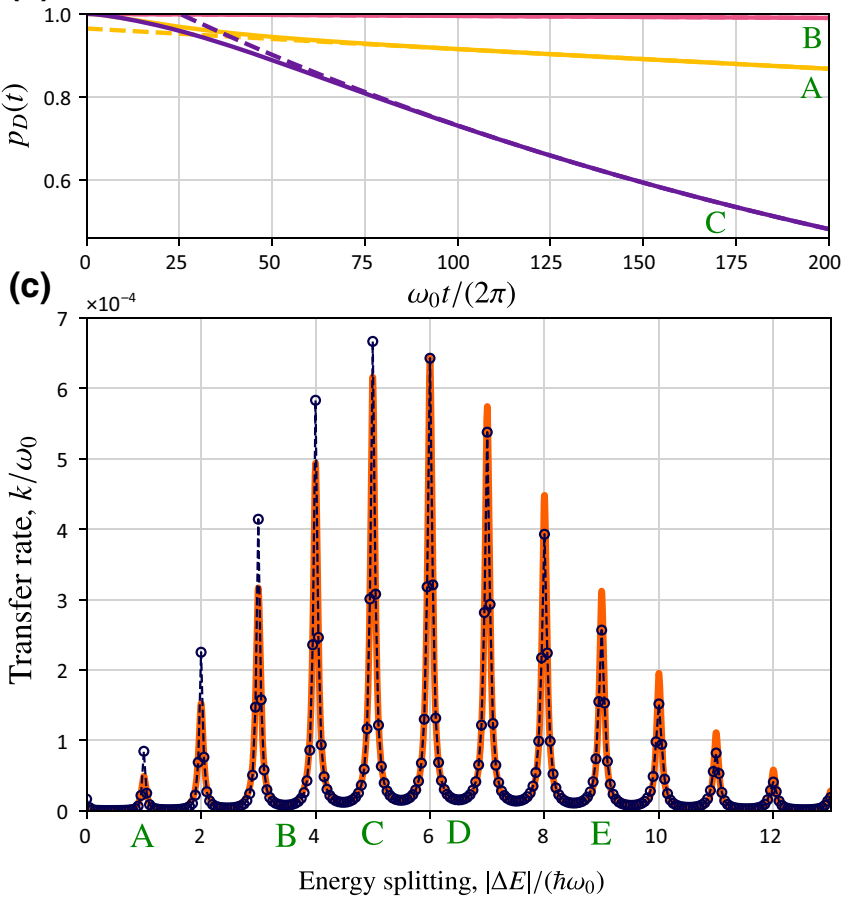

FIG. 3. Quantum nonadiabatic (QN) transfer. (a) Diabatic BO energy surfaces and their vibrational levels for the quantum nonadiabatic transfer regime. The system parameters are set to $V=0.01 \hbar \omega_{0}, g=2.5 \omega_{0}, \gamma=0.05 \omega_{0} / 2 \pi$, and $\bar{n}=0.01$. Consequently, the initial thermal population beyond the ground state amounts to less than $1 \%$, corresponding to $k_{B} T \approx 0.217 \hbar \omega_{0}$ and we further have $\lambda=6.25 \hbar \omega_{0}$. The panels show $|\Delta E|=\hbar \omega_{0}(\mathrm{~A})$, $|\Delta E|=3.5 \hbar \omega_{0}$ (B), $|\Delta E|=5 \hbar \omega_{0}(\mathrm{C}),|\Delta E|=6.5 \hbar \omega_{0}(\mathrm{D})$, and $|\Delta E|=9 \hbar \omega_{0}$ (E). (b) The dynamical evolution of the donor population (continuous lines), with exponential fits (dashed lines), for the parameters of panels A (yellow), B (pink), and C (violet). (c) The transfer rates (dark blue circles) are extracted from the exponential fits in (b). The orange line displays the prediction given in Eq. (5) of the quantum nonadiabatic theory, with resonances of finite width determined by the cooling rate $\gamma$.

removed by dissipation (implemented by the cooling laser with rate $\gamma$ ). In the above limit, however, the population is transferred back and forth between the donor and the acceptor many times before dissipation renders the process irreversible. Consequently, $\gamma$ becomes the limiting factor that controls the transfer rate and a simple model restricted to only the initial state $|D\rangle|0\rangle$ (corresponding to the zero-temperature limit) and a single resonant vibronic state $|A\rangle|v\rangle$ yields a transfer rate of (see Appendix B)

$$
k_{\mathrm{QA}}^{0 v} \propto v \gamma
$$



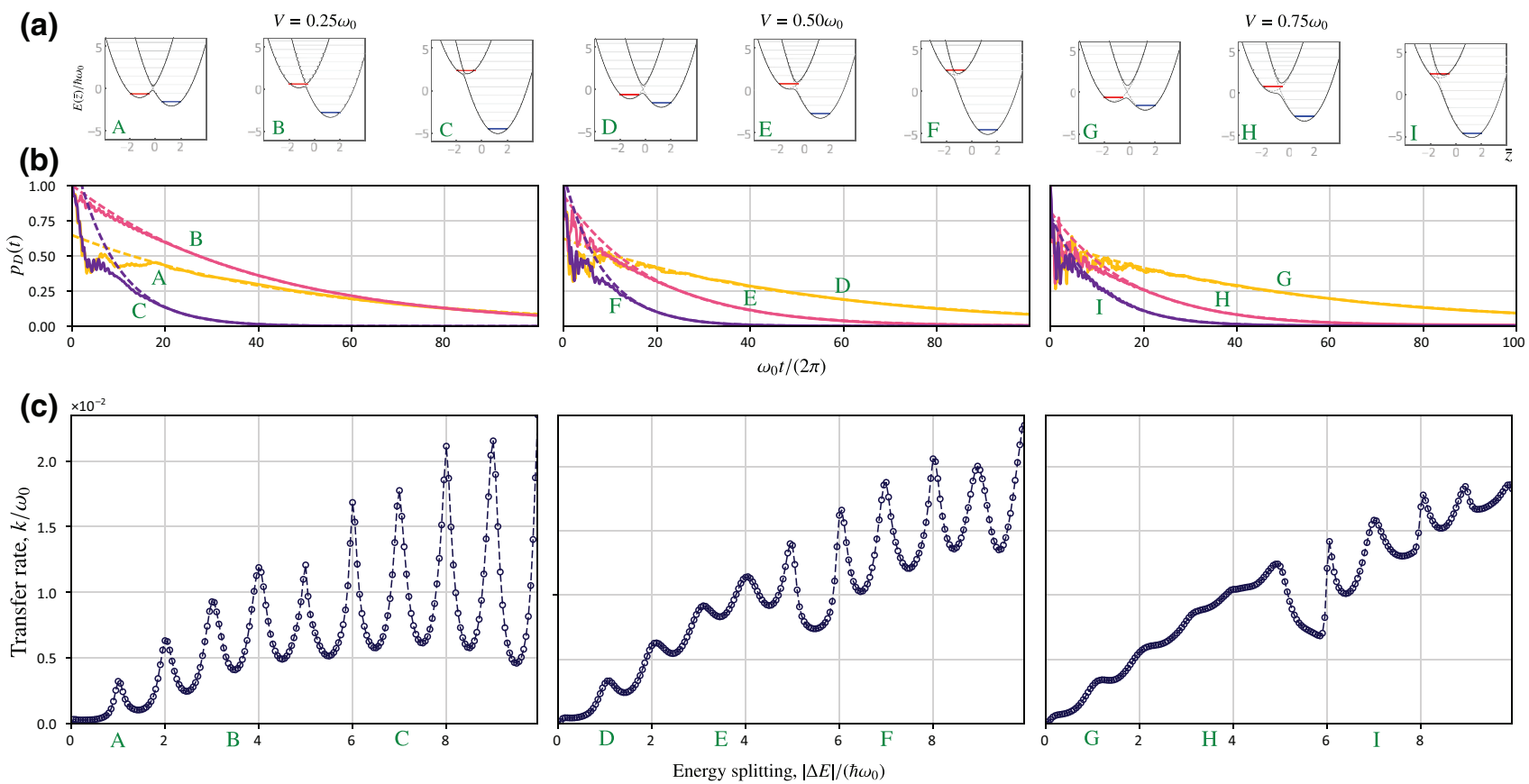

FIG. 4. The crossover toward quantum adiabatic transfer and population trapping. (a) The BO surfaces split into lower and upper adiabatic surfaces, separated by an avoided crossing of size $2|V|$. The diabatic picture is no longer adequate due to the formation of hybridized vibronic states. (b) The time evolution of the donor-state population as generated by Eqs. (1) and (3), for $V=0.25 \hbar \omega_{0}($ left panels), $V=0.50 \hbar \omega_{0}$ (center), and $V=0.75 \hbar \omega_{0}$ (right). All remaining parameters are chosen as in Fig. 3 . The plots show the evolution at $|\Delta E|=\hbar \omega_{0}$ (A,D,G; yellow lines), $|\Delta E|=3.5 \hbar \omega_{0}$ (B,E,H; pink lines), and $|\Delta E|=7 \hbar \omega_{0}$ (C,F,I; violet lines). The corresponding dashed lines show exponential fits after a transient evolution time of $\omega_{0} t / 2 \pi=20$. (c) The transfer rates extracted from the exponential fits of the simulated quantum dynamics, as a function of the donor-acceptor energy gap $\Delta E$.

which is simply the decay rate of the $v$ th vibrational level, according to the dissipator given in Eq. (3). This regime of transfer rates limited by $\gamma$, where the direct coupling $V$ no longer affects the transfer rate as in Eq. (5), is not readily accessible in a condensed-phase environment where vibrational relaxation is fast. Equation (6) neglects coupling to off-resonant states, which becomes important when the direct coupling $V$ approaches the phonon energy $\hbar \omega_{0}$ (leading to avoided crossings with widths on the order of phonon energies), as well as the effect of $V$ on the phonon lifetime due to hybridization of vibrational levels near resonance. Nevertheless, it allows us to understand the basic transport mechanism as mediated by the vibrational excitations of the emerging adiabatic BO ground state - which can therefore be considered as a quantum adiabatic (QA) transfer regime.

We show examples of such quantum adiabatic time evolution in Fig. 4(b) and the variation of the transfer rate as a function of the donor-acceptor energy gap $\Delta E$ in Fig. 4(c), with the same parameters as in Fig. 3, except for the much larger electronic coupling strengths $\left[V /\left(\hbar \omega_{0}\right)=\right.$ $0.25,0.50,0.75]$. Whereas in Fig. 3 the transfer rate is limited by the Franck-Condon factors of the transition from $v=0$ to $v^{\prime}$ phonons [see Eq. (5)] and thus peaks at $|\Delta E|=5 \hbar \omega_{0}$ (the classically activationless case), here the adiabatic rate is merely limited by the cooling rate and on resonance keeps increasing with $\Delta E$. In the normal transfer regime $\left(|\Delta E|<5 \hbar \omega_{0}\right)$, the resonances broaden with increasing direct coupling $V$ and the transfer rates eventually converge to the adiabatic limit given in Eq. (6) [see the yellow and pink lines in Fig. 5(a)]. The drivinginduced broadening of the resonances induces the rate to shrink at resonance as $V$ is further increased, while the offresonant rates grow, which explains the slight decrease at very large values of $V$. Furthermore, Fig. 5(b) demonstrates that $k / \gamma$ scales approximately linearly with $v$, as predicted by Eq. (6).

Yet another structural feature comes into play in the inverted regime $\left(|\Delta E|>5 \hbar \omega_{0}\right)$ : As is apparent in particular in the limit of large $V\left[\geq 0.50 \omega_{0}\right.$ in Fig. 4(c)], the transfer resonances broaden less strongly and, incidentally, sharpen asymmetrically. Furthermore, the transfer rates do not saturate with increasing $V$, as is to be expected from Eq. (6), but instead decay after reaching a maximum for an optimal value of the coupling [see Fig. 5(a)]. Closer inspection of the spectral structure of the Hamiltonian (1) indeed reveals that the dominant eigenstate in the time evolution of the initial state selected here (approximately $|D\rangle|0\rangle)$ is trapped and strongly localized in the excited adiabatic BO surface. In contrast to the population 

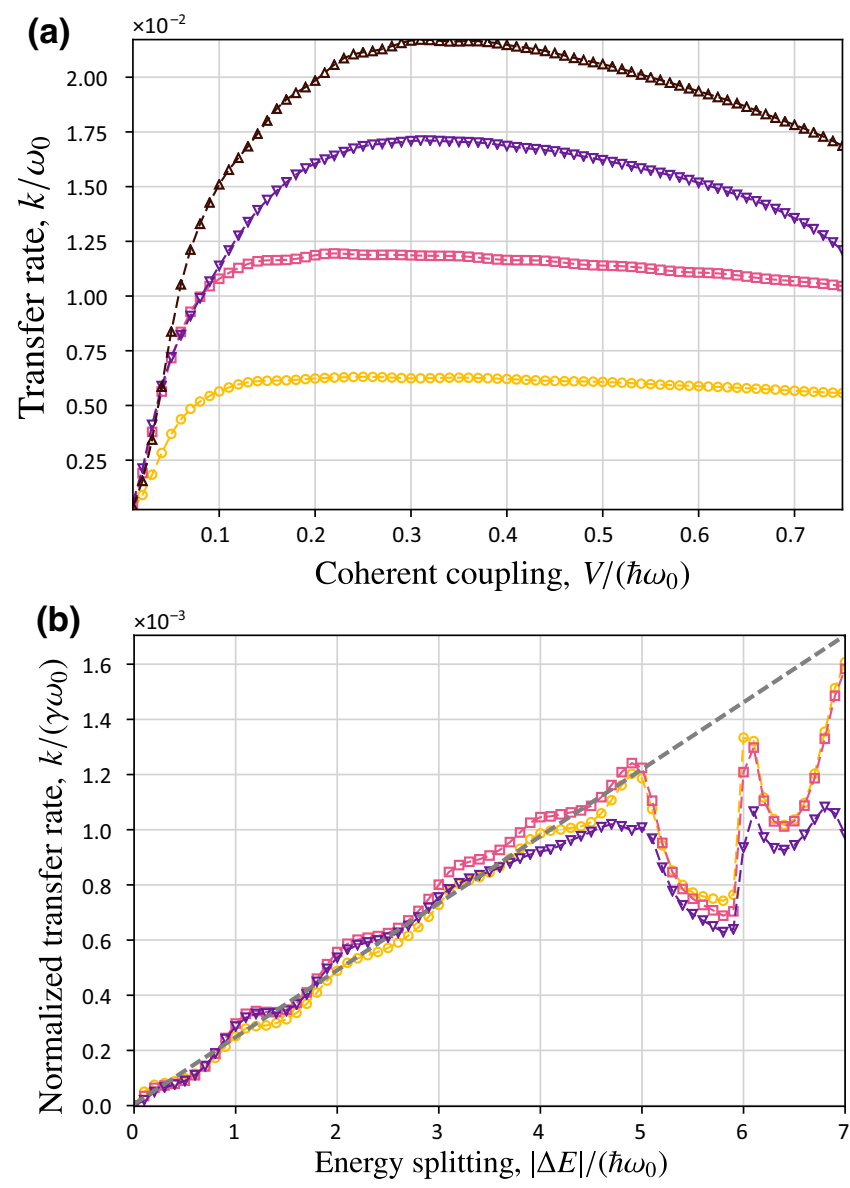

FIG. 5. The properties of the transfer rate in the quantum adiabatic limit. (a) The quantum ET rates as a function of the electronic coupling $V$ for $|\Delta E|=2 \hbar \omega_{0}$ (yellow circles), $|\Delta E|=$ $4 \hbar \omega_{0}$ (pink squares), $|\Delta E|=6 \hbar \omega_{0}$ (violet lower triangles), and $|\Delta E|=8 \hbar \omega_{0}$ (black upper triangles). (b) The quantum ET rates divided by the cooling rate $\gamma$, as a function of the donor-acceptor energy gap $\Delta E$, for $V=0.75 \hbar \omega_{0}$ and $\gamma=0.025 \omega_{0} / 2 \pi$ (yellow circles), $\gamma=0.05 \omega_{0} / 2 \pi$ (pink squares), and $\gamma=0.075 \omega_{0} / 2 \pi$ (violet lower triangles). The rates in the normal regime $(|\Delta E|<$ $\left.5 \hbar \omega_{0}\right)$ approximately follow the quantum adiabatic limit given in Eq. (6), which predicts a linear dependence on $|\Delta E|$ (since the latter controls the resonantly coupled phonon number $v$ ). For $|\Delta E|>5 \hbar \omega_{0}$, the process is no longer purely quantum adiabatic due to the trapping of part of the initial population in the upper adiabatic BO surface (see the text). The dashed line shows a linear fit, $a|\Delta E| /\left(\hbar \omega_{0}\right)+b$, with $a=2.4 \times 10^{-4} \gamma \omega_{0}$ and $b=5.2 \times 10^{-6} \gamma \omega_{0}$. All remaining parameters are chosen as in Fig. 3.

on the lower BO surface, the excited population remains trapped unless transfer is facilitated by a resonance, as we can clearly observe in Fig. 4(c) for $V=0.75 \omega_{0}$ (see Appendix 3). These observations imply that experimental control over the initial state, which can be achieved with high accuracy in trapped-ion experiments, defines yet another handle to control the ET efficiency in this largely unexplored parameter regime.

\section{POSSIBLE EXTENSIONS OF THE SETUP}

In this paper, we study ET simulations involving only a single vibrational mode. In many molecular ET reactions in condensed-phase environments, a large number $N_{\text {vib }}$ of vibrational modes $\mu$ are ET active, with $\lambda_{\mu} / \hbar \omega_{\mu}>1$ and $k_{B} T / \hbar \omega_{\mu} \geq 1$. Quantum effects play a relevant role in the dynamics of such modes but their exact treatment is usually intractable [57]. Instead, numerical ET simulations use approximations that treat a large fraction of ET-active modes as classical vibrations. Trapped-ion quantum simulations provide an alternative to large-scale numerical simulations and semiclassical approximations, by reproducing the fully quantum ET dynamics in a well-controlled setup. Experimental quantum simulations of ET may thus complement theoretical, computational, and experimental efforts on molecular ET systems and may be used to benchmark approximate models. This opens up new pathways toward the microscopic understanding of ET processes, the identification of novel transfer regimes, and the design of highly efficient ET systems.

Possible extensions of our proposal include coherent coupling to multiple motional modes to enable vibronic transfer on a more complex vibrational backbone or to study the influence of intermediate bridge states [48]. This could be implemented by increasing the number of ions and inducing laser coupling that encompass multiple motional modes [44]. More flexibility in tuning the electronic transfer properties of the system is offered by engineered electronic coupling between different ions with a tunable interaction range, which can be controlled via an effective spin-spin coupling using additional motional modes $[58,59]$. These methods can be combined with two-dimensional traps with particular lattice geometries $[47,60]$. Other studies may target the influence of different initial states, including nonequilibrium or even nonclassical vibrations $[15,52,61-63]$.

\section{CONCLUSIONS}

We show that trapped-ion analog quantum simulators provide a versatile test bed for studying phonon-mediated electron transfer under well-controlled adjustable conditions. Numerical simulations demonstrate that a rather simple system composed of two ions coupled to a single phononic mode is able to reproduce the essential features of donor-acceptor molecular ET. Continuously tuning the parameters allows us to connect and study a great variety of largely unexplored transport regimes that are not readily accessed by molecular ET experiments in a continuous way. When operated in a high-temperature regime, the predictions of Marcus theory can be verified for classical nonadiabatic ET, with a high level of control over individual parameters. In the low-temperature regime, it is possible to study the emergence of quantum nonadiabatic vibronic transfer resonances. By further increasing 
the electronic coupling, quantum adiabatic transport is observed only in the normal regime, whereas the inverted regime gives rise to a transfer mechanism mediated by resonances due to trapped excited-state populations. It is impossible to realize such a controlled crossover between adiabatic and nonadiabatic regimes in molecular systems.

Our work points out that dissipation and incoherent features in the long-time dynamics of quantum simulators can give rise to interesting transport phenomena that are of high relevance, e.g., in molecular chemistry. These features become visible in the decay rates after the coherent phenomena have largely been damped out by the engineered dissipation of phonons. Our approach is therefore complementary to predominantly coherent quantum simulations of the short-time dynamics that provide access to strongly entangled many-body systems by trying to mitigate decoherence $[23,24]$. At longer time scales, the natural decoherence of the trapped-ion system becomes increasingly relevant. However, the time evolutions considered here are on the order of few milliseconds. This is still shorter than realistic quantum error correction cycles [27], which in turn are subject to much stronger restrictions in terms of fidelity and coherence than our simulations.

\section{ACKNOWLEDGMENTS}

A.B., T.S., and S.S.S. thank the Freiburg Institute of Advanced Studies (FRIAS) for support of the present cooperation through the Research Focus "Designed Quantum Transport in Complex Materials." All of the authors thank A. Bermudez, H.-P. Breuer, and H. Haeffner for useful discussions. F.S. acknowledges funding from the European Research Council under the European Union's Seventh Framework Programme (FP7/2007-2013) Grant Agreement No. 319286 Q-MAC. M.G. acknowledges support by the LabEx ENS-ICFP under Grants No. ANR-10LABX-0010 and No. ANR-10-IDEX-0001-02 PSL*. T.S. acknowledges support from the Deutsche Forschungsgemeinschaft under Grant No. SCHA 973. S.S.S. thanks the People Programme (Marie Curie Actions) of the European Union's Seventh Framework Programme (Grant No. FP7/2007-2013), under the Research Executive Agency Grants No. 609305, and FRIAS for hosting him as an External Senior Fellow. The numerical simulations were performed with the aid of the QuTIP package for PYTHON [64].

F. Schlawin and M. Gessner contributed equally to this work.

\section{APPENDIX A: DETERMINATION OF THE DECAY RATE}

The information about the decay rates is contained in the long-time dynamics of the ion-trap system. In our simulations, these are obtained by propagating for sufficiently long times. Alternatively, if it is hard to extract the decay constant by an exponential fit (e.g., due to shorter propagation times), the decay rate can be efficiently approximated using [65]

$$
k^{-1}=\frac{\int d t t p_{D}(t)}{\int d t p_{D}(t)}
$$

where the integral excludes a transient initial time interval that is dominated by coherent effects. In the case of a pure exponential decay $\exp (-k t)$, the decay rate exactly reduces to the decay constant $k$.

\section{APPENDIX B: QUANTUM ADIABATIC DYNAMICS}

To understand the transition from the Fermi golden rule result given in Eq. (5) to stronger interaction strengths $V$ in the quantum transport regime, we consider more closely the resonance between the initial state $|D\rangle|0\rangle$ and an acceptor state $|A\rangle|\nu\rangle$. Such a resonance is achieved for $v=|\Delta E| /\left(\hbar \omega_{0}\right)$ [see Fig. 1(a)]. The decay of the excited state can be described by the effective non-Hermitian Hamiltonian

$$
H=\left(\begin{array}{cc}
0 & V \sqrt{\mathrm{FC}_{0, v}} \\
V^{*} \sqrt{\mathrm{FC}_{0, v}} & -i \tilde{\gamma}
\end{array}\right),
$$

where $\tilde{\gamma}$ is the decay rate of the acceptor state. Here, we assume that the electronic coupling $V$ is further modulated by the Franck-Condon factor $\mathrm{FC}_{0, v}$ and we neglect the coupling to other states. The donor population decays as

$$
p_{D}(t)=e^{-\tilde{\gamma} t}\left[\cosh \left(\frac{d t}{2}\right)+\frac{\tilde{\gamma}}{d} \sinh \left(\frac{d t}{2}\right)\right]^{2},
$$

with $d=\sqrt{\tilde{\gamma}^{2}-4|V|^{2} \mathrm{FC}_{0, v}}$. This can be mapped to the Jaynes-Cummings model on resonance, with a cavity mode that is coupled to a reservoir, leading to a Lorentzian spectral density [33]. The insertion of Eq. (B2) into Eq. (A1) yields the decay rate

$$
k_{\mathrm{QA}}^{0 v}=\tilde{\gamma} \frac{1+\eta^{2}}{1+\frac{1}{2} \eta^{4}},
$$

where the parameter $\eta=\tilde{\gamma} /\left(|V| \sqrt{\mathrm{FC}_{0, v}}\right)$ determines the ratio of the phonon decay rate and the coherent coupling strength.

\section{Weak coupling}

The weak-coupling limit, $|V| \sqrt{\mathrm{FC}_{0, v}} \ll \tilde{\gamma}$, is characterized by $\eta \gg 1$. We obtain

$$
k_{\mathrm{QA}}^{0 \nu}=\frac{2|V|^{2} \mathrm{FC}_{0, v}}{\tilde{\gamma}}+\mathcal{O}\left(\tilde{\gamma} \eta^{-4}\right)
$$


With $\tilde{\gamma}=\gamma$, this reproduces the Marcus result pertaining to the one transfer channel on resonance and the delta function broadened to a Lorentzian with width $\gamma$. It coincides with the nonadiabatic result given in Eq. (5) on resonance.

\section{Strong coupling}

In the opposite limit, $\eta \ll 1$, we obtain

$$
k_{\mathrm{QA}}^{0 v}=\tilde{\gamma}+\mathcal{O}\left(\tilde{\gamma} \eta^{2}\right),
$$

leading to Eq. (6) for $\tilde{\gamma}=v \gamma$, i.e., the decay rate of the $v$ th phononic level.

\section{Eigenstate width in the inverted regime}

To understand the survival of the sharp resonances in the inverted regime, we consider the "eigenstate width" [66] of the initial state

$$
W=\exp \left(-\sum_{j} p_{j} \ln p_{j}\right),
$$

where $p_{j} \equiv\langle j|\rho| j\rangle$ is the overlap of the initial density matrix $\rho=|D\rangle\left\langle D\left|\otimes \sum_{\nu} p_{v}\right| v\right\rangle\langle v|$ with the eigenstate $|j\rangle$ of the full Hamiltonian (1) and we use the convention $p \ln p=0$ for $p=0$. Intuitively, a larger value of $W$ indicates an increasingly complex dynamical evolution that, in a superposition of many different energy eigenstates, explores a larger fraction of the state space. This increases the likelihood of populating states that respond strongly to the laser-cooling process and thus lead to an irreversible transfer of population from $|D\rangle \rightarrow|A\rangle$.

The width [see Eq. (B6)] is shown in Fig. 6 for three different interaction strengths $V$, corresponding to the three

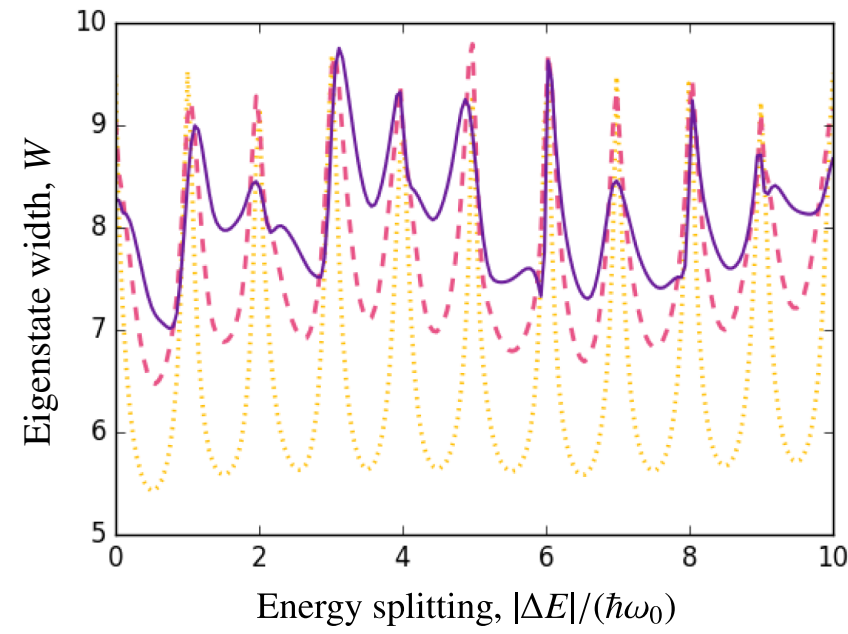

FIG. 6. The eigenstate width of the initial state according to Eq. (B6) for $V=0.25 \hbar \omega_{0}$ (yellow, dot-dashed), $0.5 \hbar \omega_{0}$ (red, dashed), and $0.75 \hbar \omega_{0}$ (blue, solid line). All remaining parameters are identical to those in Fig. 4. panels in Fig. 4. We observe a clear resonance structure that is less and less pronounced as the interaction strength is increased. However, the broadening arises asymmetrically: while the resonances are strongly washed out in the range $\Delta E / \hbar \omega_{0}=3, \ldots, 5$, the eigenstate width remains more clearly peaked at integer values of $\Delta E / \hbar \omega_{0}$. In particular, the resonances at $\Delta E / \hbar \omega_{0}=6$ and 8 remain very sharp, reflecting the behavior of the transfer rate in the simulation in Fig. 4(c). Resonances at $\Delta E / \hbar \omega_{0}=$ 7 and 9 are broadened more strongly, again, in agreement with the transfer-rate simulations. In summary, the analysis of the eigenstate width hence reveals (a) that in the inverted regime, efficient transport $|D\rangle \rightarrow|A\rangle$ relies on the resonance of diabatic states, and (b) that the shape of the resonances is related to the distribution of energy eigenstates in the initial density matrix.

[1] R. A. Marcus and N. Sutin, Electron transfers in chemistry and biology, Biochim. Biophys. Acta 811, 265 (1985).

[2] A. M. Kuznetsov and J. Ulstrup, Electron Transfer in Chemistry and Biology: An Introduction to the Theory (Wiley, Hoboken, NJ, USA, 1999).

[3] J. Jortner and M. Bixon, Electron Transfer: From Isolated Molecules to Biomolecules, Part 1, edited by I. Prigogine and S. A. Rice, Advances in Chemical Physics Vol. 106 (Wiley, 1999), p. 35.

[4] V. May and O. Kühn, Charge and Energy Transfer Dynamics in Molecular Systems (Wiley-VCH, Berlin, 2000).

[5] V. Balzani (Ed.), Electron Transfer in Chemistry (WileyVCH, Weinheim, 2001).

[6] A. Nitzan, Chemical Dynamics in Condensed Phases (Oxford University Press, Oxford, UK, 2006).

[7] R. E. Blankenship, Molecular Mechanisms of Photosynthesis (Blackwell Science, Oxford, UK, 2002).

[8] M. Mohseni, Y. Omar, G. Engel, and M. Plenio (Eds.), Quantum Effects in Biology (Cambridge University Press, Cambridge, UK, 2014).

[9] J. C. Cuevas and E. Scheer, Molecular Electronics: An Introduction to Theory and Experiment (World Scientific, Singapore, 2017), 2nd ed.

[10] G. D. Scholes, G. R. Fleming, L. X. Chen, A. AspuruGuzik, A. Buchleitner, D. F. Coker, G. S. Engel, R. van Grondelle, A. Ishizaki, D. M. Jonas, J. S. Lundeen, J. K. McCusker, S. Mukamel, J. P. Ogilvie, A. Olaya-Castro, M. A. Ratner, F. C. Spano, K. B. Whaley, and X. Zhu, Using coherence to enhance function in chemical and biophysical systems, Nature 543, 647 (2017).

[11] D. N. Beratan, S. S. Skourtis, T. J. Erb, and J. A. Gerlt (Eds.), Energy/Mechanistic Biology, Current Opinion in Chemical Biology (Elsevier, Amsterdam, 2018), Vol. 47.

[12] S. S. Skourtis, D. H. Waldeck, and D. N. Beratan, Fluctuations in biological and bioinspired electron-transfer reactions, Annu. Rev. Phys. Chem. 61, 461 (2010).

[13] J. Blumberger, Recent advances in the theory and molecular simulation of biological electron transfer reactions, Chem. Rev. 115, 11191 (2015). 
[14] D. N. Beratan, C. Liu, A. Migliore, N. F. Polizzi, S. S. Skourtis, P. Zhang, and Y. Zhang, Charge transfer in dynamical biosystems, or the treachery of (static) images, Acc. Chem. Res. 48, 474 (2015).

[15] S. Valianti and S. S. Skourtis, Vibrational control of molecular electron transfer reactions, Mol. Phys. 117, 2618 (2018).

[16] S. Rafiq and G. D. Scholes, From fundamental theories to quantum coherences in electron transfer, J. Am. Chem. Soc. 141, 708 (2019).

[17] J. R. Winkler and H. B. Gray, Electron flow through metalloproteins, Chem. Rev. 114, 3369 (2014).

[18] J. R. Miller, L. T. Calcaterra, and G. L. Closs, Intramolecular long-distance electron transfer in radical anions. The effects of free energy and solvent on the reaction rates, J. Am. Chem. Soc. 106, 3047 (1984).

[19] G. L. Closs and J. R. Miller, Intramolecular long-distance electron transfer in organic molecules, Science 240, 440 (1988).

[20] R. A. Marcus, Electron transfer reactions in chemistry: Theory and experiment (Nobel Lecture), Angew. Chem. Int. Ed. Engl. 32, 1111 (1993).

[21] R. Blatt and D. Wineland, Entangled states of trapped atomic ions, Nature 453, 1008 (2008).

[22] H. Häffner, C. F. Roos, and R. Blatt, Quantum computing with trapped ions, Phys. Rep. 469, 155 (2008).

[23] C. Schneider, D. Porras, and T. Schaetz, Experimental quantum simulations of many-body physics with trapped ions, Rep. Prog. Phys. 75, 024401 (2012).

[24] R. Blatt and C. Roos, Quantum simulations with trapped ions, Nat. Phys. 8, 277 (2012).

[25] J.-S. Chen, S. M. Brewer, C. W. Chou, D. J. Wineland, D. R. Leibrandt, and D. B. Hume, Sympathetic Ground State Cooling and Time-Dilation Shifts in an ${ }^{27} \mathrm{Al}^{+}$Optical Clock, Phys. Rev. Lett. 118, 053002 (2017).

[26] T. H. Johnson, S. R. Clark, and D. Jaksch, What is a quantum simulator? EPJ Quantum Technol. 1, 10 (2014).

[27] A. Bermudez, X. Xu, R. Nigmatullin, J. O'Gorman, V. Negnevitsky, P. Schindler, T. Monz, U. G. Poschinger, C. Hempel, J. Home, F. Schmidt-Kaler, M. Biercuk, R. Blatt, S. Benjamin, and M. Müller, Assessing the Progress of Trapped-Ion Processors Towards Fault-Tolerant Quantum Computation, Phys. Rev. X 7, 041061 (2017).

[28] A. Bermudez, M. Bruderer, and M. B. Plenio, Controlling and Measuring Quantum Transport of Heat in Trapped-Ion Crystals, Phys. Rev. Lett. 111, 040601 (2013).

[29] M. Ramm, T. Pruttivarasin, and H. Häffner, Energy transport in trapped ion chains, New J. Phys. 16, 063062 (2014).

[30] A. Abdelrahman, O. Khosravani, M. Gessner, A. Buchleitner, H.-P. Breuer, D. Gorman, R. Masuda, T. Pruttivarasin, M. Ramm, P. Schindler, and H. Häffner, Local probe of single phonon dynamics in warm ion crystals, Nat. Comm. 8, 15712 (2017).

[31] A. Bermudez and T. Schaetz, Quantum transport of energy in controlled synthetic quantum magnets, New J. Phys. 18, 083006 (2016).

[32] D. J. Gorman, B. Hemmerling, E. Megidish, S. A. Moeller, P. Schindler, M. Sarovar, and H. Haeffner, Engineering Vibrationally Assisted Energy Transfer in a Trapped-Ion Quantum Simulator, Phys. Rev. X 8, 011038 (2018).
[33] H.-P. Breuer and F. Petruccione, The Theory of Open Quantum Systems (Oxford University Press, Oxford, UK, 2007).

[34] D. Leibfried, R. Blatt, C. Monroe, and D. Wineland, Quantum dynamics of single trapped ions, Rev. Mod. Phys. 75, 281 (2003).

[35] D. J. Larson, J. C. Bergquist, J. J. Bollinger, W. M. Itano, and D. J. Wineland, Sympathetic Cooling of Trapped Ions: A Laser-Cooled Two-Species Nonneutral Ion Plasma, Phys. Rev. Lett. 57, 70 (1986).

[36] B. B. Blinov, L. Deslauriers, P. Lee, M. J. Madsen, R. Miller, and C. Monroe, Sympathetic cooling of trapped $\mathrm{Cd}^{+}$isotopes, Phys. Rev. A 65, 040304(R) (2002).

[37] M. D. Barrett, B. DeMarco, T. Schaetz, V. Meyer, D. Leibfried, J. Britton, J. Chiaverini, W. M. Itano, B. Jelenković, J. D. Jost, C. Langer, T. Rosenband, and D. J. Wineland, Sympathetic cooling of ${ }^{9} \mathrm{Be}^{+}$and $24 \mathrm{Mg}^{+}$ for quantum logic, Phys. Rev. A 68, 042302 (2003).

[38] M. Drewsen, A. Mortensen, R. Martinussen, P. Staanum, and J. L. Sørensen, Nondestructive Identification of Cold and Extremely Localized Single Molecular Ions, Phys. Rev. Lett. 93, 243201 (2004).

[39] J. P. Home, D. Hanneke, J. D. Jost, J. M. Amini, D. Leibfried, and D. J. Wineland, Complete methods set for scalable ion trap quantum information processing, Science 325, 1227 (2009).

[40] S. Willitsch, Coulomb-crystallised molecular ions in traps: Methods, applications, prospects, Int. Rev. Phys. Chem. 31, 175 (2012).

[41] D. F. V. James, Quantum dynamics of cold trapped ions with application to quantum computation, Appl. Phys. B 66, 181 (1998).

[42] K. Kim, C. F. Roos, L. Aolita, H. Häffner, V. Nebendahl, and R. Blatt, Geometric phase gate on an optical transition for ion trap quantum computation, Phys. Rev. A 77, 050303(R) (2008).

[43] D. Porras, F. Marquardt, J. Delft, and J. I. Cirac, Mesoscopic spin-boson models of trapped ions, Phys. Rev. A 78, 010101 (2008).

[44] G. Clos, D. Porras, U. Warring, and T. Schätz, TimeResolved Observation of Thermalization in an Isolated Quantum System, Phys. Rev. Lett. 117, 170401 (2016).

[45] A. Lemmer, C. Cormick, D. Tamascelli, T. Schaetz, S. F. Huelga, and M. B. Plenio, A trapped-ion simulator for spinboson models with structured environments, New J. Phys. 20, 073002 (2018).

[46] D. Lv, S. An, Z. Liu, J.-J. Zhang, J. S. Pedernales, L. Lamata, E. Solano, and K. Kim, Quantum Simulation of the Quantum Rabi Model in a Trapped Ion, Phys. Rev. X 8, 021027 (2018).

[47] F. Hakelberg, P. Kiefer, M. Wittemer, U. Warring, and T. Schaetz, Interference in a Prototype of a TwoDimensional Ion Trap Array Quantum Simulator, Phys. Rev. Lett. 123, 100504 (2019).

[48] S. S. Skourtis and D. N. Beratan, Theories of structurefunction relationships for bridge-mediated electron transfer reactions, Adv. Chem. Phys. 106, 377 (1999).

[49] C. Langer, R. Ozeri, J. D. Jost, J. Chiaverini, B. DeMarco, A. Ben-Kish, R. B. Blakestad, J. Britton, D. B. Hume, W. M. Itano, D. Leibfried, R. Reichle, T. Rosenband, 
T. Schaetz, P. O. Schmidt, and D. J. Wineland, Long-Lived Qubit Memory Using Atomic Ions, Phys. Rev. Lett. 95, 060502 (2005).

[50] S. Haze, Y. Tateishi, A. Noguchi, K. Toyoda, and S. Urabe, Observation of phonon hopping in radial vibrational modes of trapped ions, Phys. Rev. A 85, 031401(R) (2012).

[51] R. Lechner, C. Maier, C. Hempel, P. Jurcevic, B. P. Lanyon, T. Monz, M. Brownnutt, R. Blatt, and C. F. Roos, Electromagnetically-induced-transparency groundstate cooling of long ion strings, Phys. Rev. A 93, 053401 (2016).

[52] J. F. Poyatos, J. I. Cirac, and P. Zoller, Quantum Reservoir Engineering with Laser Cooled Trapped Ions, Phys. Rev. Lett. 77, 4728 (1996).

[53] C. Cormick, A. Bermudez, S. F. Huelga, and M. B. Plenio, Dissipative ground-state preparation of a spin chain by a structured environment, New J. Phys. 15, 073027 (2013).

[54] C. J. Myatt, B. E. King, Q. A. Turchette, C. A. Sackett, D. Kielpinski, W. M. Itano, C. Monroe, and D. J. Wineland, Decoherence of quantum superpositions through coupling to engineered reservoirs, Nature 403, 269 (2000).

[55] S. Sumi, in Electron Transfer in Chemistry, edited by V. Balzani (Wiley-VCH, Weinheim, 2001), p. 65.

[56] To account for the line broadening due to the sympathetic cooling, given in Eq. (3), we replace the delta function in Eq. (5) by a normalized Lorentzian frequency distribution of width $2 \pi \hbar \gamma$, i.e., $\delta\left(E_{D}, v-E_{A}, v^{\prime}\right) \longrightarrow 2 \hbar \gamma /\left(\left(E_{D}, v-\right.\right.$ $\left.\left.E_{A}, v^{\prime}\right)^{2}+(2 \pi \hbar \gamma)^{2}\right)$. The slight shift between the theory prediction and the simulation stems from an additional $\Delta E$ dependent broadening, which cannot be resolved in Fig. 3 and that enhances the broadening of resonances in the inverted regime.
[57] In fully quantum modeling, a very large number of phononexcited states per mode $\left(n_{\mu} \gg \lambda_{\mu} / \hbar \omega_{\mu},|\Delta E| / \hbar \omega_{\mu}\right.$ and $\left.k_{B} T / \hbar \omega_{\mu}\right)$ would have to be included in the vibronic state space to achieve accuracy (i.e., $N_{\text {vib }} \times n_{\mu}$ vibrational states for each electronic state).

[58] A. Sørensen and K. Mølmer, Quantum Computation with Ions in Thermal Motion, Phys. Rev. Lett. 82, 1971 (1999).

[59] D. Porras and J. I. Cirac, Effective Quantum Spin Systems with Trapped Ions, Phys. Rev. Lett. 92, 207901 (2004).

[60] P. Kiefer, F. Hakelberg, M. Wittemer, A. Bermúdez, D. Porras, U. Warring, and T. Schaetz, Floquet-Engineered Vibrational Dynamics in a Two-Dimensional Array of Trapped Ions, Phys. Rev. Lett. 123, 213605 (2019).

[61] D. M. Meekhof, C. Monroe, B. E. King, W. M. Itano, and D. J. Wineland, Generation of Nonclassical Motional States of a Trapped Atom, Phys. Rev. Lett. 76, 1796 (1996).

[62] F. Wolf, C. Shi, J. C. Heip, M. Gessner, L. Pezzè, A. Smerzi, M. Schulte, K. Hammerer, and P. O. Schmidt, Motional Fock states for quantum-enhanced amplitude and phase measurements with trapped ions, Nat. Commun. 10, 2929 (2019).

[63] C. Flühmann, T. L. Nguyen, M. Marinelli, V. Negnevitsky, K. Mehta, and J. P. Home, Encoding a qubit in a trapped-ion mechanical oscillator, Nature 566, 513 (2019).

[64] J. Johansson, P. Nation, and F. Nori, QuTiP 2: A PYTHON framework for the dynamics of open quantum systems, Comput. Phys. Commun. 184, 1234 (2013).

[65] S. S. Skourtis, A. J. R. Da Silva, W. Bialek, and J. N. Onuchic, New look at the primary charge separation in bacterial photosynthesis, J. Phys. Chem. 96, 8034 (1992).

[66] R. Blümel and U. Smilansky, Microwave ionization of highly excited hydrogen atoms, Z. Phys. D 6, 83 (1987). 